\title{
Disección endoscópica de la submucosa (DES) vs. disección endoscópica de espesor total (DET) para el tratamiento de cáncer de colon
}

\author{
Oscar V. Hernández-Mondragón* y Raúl A. Zamarripa-Mottú \\ Departamento de Endoscopia gastrointestinal, Hospital de Especialidades Dr. Bernardo Sepúlveda Gutiérrez, Centro Médico Nacional Siglo XXI, \\ Ciudad de México, México
}

\begin{abstract}
Resumen
Existen 4 modalidades de tratamiento endoscópico para tratamiento de cáncer colorectal: la resección endoscópica de la mucosa (REM) es eficaz pero limitada a la mucosa ${ }^{1}$; la disección endoscópica de la submucosa (DES) y la disección endoscópica de espesor total (DET) son técnicas de resección endoscópica avanzadas que pueden tratar lesiones recurrentes post-REM (20\%) o no candidatas a la misma² y, finalmente, la disección endoscópica híbrida (DEH) o una combinación de las 3 técnicas mencionadas previamente, permite tratar lesiones colorrectales más grandes, sin importar si existe fibrosis, no levantan, tumores subepiteliales, carcinomas tempranos o una localización difícil (ciego, colon derecho, cerca a un divertículo o al orificio apendicular) $)^{1}$. Durante la DDW 2020 virtual se presentaron 14 trabajos relacionados, documentando los más relevantes.
\end{abstract}

Palabras clave: Disección endoscópica. Cáncer colorrectal.

\section{DES en lesiones colorrectales}

Stavropoulos $\mathrm{S}$, et al: serie más grande de DES (8 años, $n=462$ : carcinoma 42, adenoma 420, biopsia previa $432(94 \%)$, tatuaje $193(42 \%)$ y REM / ablación previa 168 (36\%), 26\% en localización difícil: sigmoides $36(8 \%)$, válvula ileocecal $25(5 \%)$, orificio apendicular $8(2 \%))$. Resección en bloque $96.5 \%$, R0 91.3\%, eventos adversos 2.4\%: perforación tardía 1, hemorragia tardía 6 , otros 2 ) y recurrencia $0.8 \%$ tras seguimiento a 1 año en $83 \%$. Limitaciones: retrospectivo ${ }^{3}$.

Lacopini $F$ et al: realizaron un ensayo clínico de DES para lesiones colorrectales $>20 \mathrm{~mm}$ o sin levantamiento de localización difícil (línea dentada $n=50$, pélvico rectal 92, invasión íleon terminal $n=27$, ciego $n=67$ y colon ascendente $n=118$ ), con cicatriz en $n=53$ / 354. Se reportó resección en bloque/ Ro/ curativa/ Residual: línea dentada $82 \% / 76 \% / 66 \% / 12 \%$ vs. pélvico rectal $89 \% / 82 \% / 82 \% / 0 \%$ vs. invasión íleon terminal $88 \% / 78 \% / 78 \% / 7 \%$ vs. ciego $83 \% / 83 \% / 56.7 \% / 3 \%$ vs. colon ascendente $90 \% / 90 \% / 85.5 \% / 1 \%, p=0.002$ para residual, $p>0.05$ para en bloque y $R 0$ ). Limitaciones: No se especifica el seguimiento 4 .

Uchima $\mathrm{H}$, et al: estudio prospectivo, multicéntrico donde evaluaron DES en pólipos malignos $(n=58$, recto $21(36 \%)$, sigmoides $10(17 \%)$, colon descendente $8(13.7 \%)$, flexura esplénica $2(3.4 \%)$, colon transverso $4(7 \%)$, flexura hepática $3(5 \%)$, Colon ascendente 7 $(12 \%)$, ciego $3(5 \%)$ con fibrosis severa $(\mathrm{F} 2)$ en 23

\section{Correspondencia:}


(39.66\%) casos. Se reportó éxito técnico en 48, resección en bloque en 41 y de estos hubo margen vertical libre $>1 \mathrm{~mm}$ en $78 \%$ (32/41), el tamaño medio de tejido resecado fue de $38.3 \mathrm{~mm} \times 30.67 \mathrm{~mm}$. La resección en bloque difiere acorde a la localización: Recto $96.3 \%$ vs. resto del colon $78.84 \%, p=0.029$. Eventos adversos en $18(31 \%)$ : hemorragia tardía $3(5 \%)$, perforaciones intraprocedimiento $10(17 \%)$ y tardías $2(3.4 \%)$. Se requirió cirugía en 30 (51.7\%): por histología 19 (63\%), por DES abortada 10 (33\%) y 1 por perforación tardía (3\%) y de estas fue menor la necesidad de cirugía en recto $n=8(38 \%)$ vs. resto de colon $n=22(59 \%)$; $p=0.029$. Limitantes: no especifican seguimiento ${ }^{5}$.

\section{DET en lesiones colorrectales}

Bazarbashi $\mathrm{A}$, et al: revisión sistemática y metaanálisis, analizaron 18 estudios (8 prospectivos, 10 retrospectivos) con un total de 757 pacientes sometidos a DET demostrando su eficacia (éxito clínico en $89.52 \%$ [86.97\%-91.62\%, IC 95\%, I2 = 0.00], resección R0 en $79.61 \%$ [75.47\%-83.2\%, IC 95\%, I2 = 20.52]) y su seguridad (eventos adversos $11.97 \%$ [hemorragia $2.31 \%$, perforación $20 \%$, síndrome pospolipectomía $1.7 \%$, apendicitis $0.8 \%$, otros $3.1 \%$, con recurrencia mayor a la observada con DES: $9.85 \%(7.26-13.23 \%$, IC $95 \%$, $12=0.00)^{6}$.

Meier B, et al: ensayo clínico multicéntrico, evaluando DET en el tratamiento de lesiones colorectales complejas, pretratadas 0 en localización difícil $n=1,178$ (colon 880 (75\%): ciego $21 \%$, colon ascendente $21 \%$, colon transverso $12 \%$, colon descendente $6 \%$, sigmoides $15 \%$ y recto 298 (25\%); lesiones pretratadas 637 (54\%)). Indicaciones: Adenoma sin levantamiento 57\%, carcinoma temprano $18 \%$, localización difícil $8.5 \%$, tumor subepitelial $7 \%$, DET diagnóstica $1.5 \%$, otros $8 \%$. Encontraron resección de espesor total global $90 \%$ (coIon $92 \%$, recto $84 \%$ ), resección R0 en $80 \%$ (colon $79 \%$, recto $82 \%$ ), tiempo de procedimiento global 35 (2-203) $\mathrm{min}$, colon 40 (3-203) min, recto 25 (2-120) min, hubo problemas técnicos en 154 (13\%): relacionados a asa 37, aplicación de clip 5 y otros en 112 (la mayoría relacionados con la movilización tisular) y eventos adversos en 138 (11.7\%): hemorragia $83(7 \%)$, perforación $29(2.5 \%)$, inflamación/infección $15(12.7 \%)$ y otros 11 $(0.93 \%)$; de los cuales $24(2 \%)$ ameritaron tratamiento quirúrgico. Recurrencia $9.4 \%$ tras seguimiento de 22 (1-202) semanas en $92 \%$ (1088/1178), con endoscopia de seguimiento en $63 \%$ (685/1088). Limitaciones: seguimiento irregular ${ }^{1}$.

\section{DES vs. DET para lesiones colorrectales}

Suchanek S, et al: ensayo clínico bicéntrico, DET vs. DES para neoplasias colorrectales $<3 \mathrm{~cm}$ con riesgo alto de resección incompleta ( $n=35$ : DET 19 vs DES 16). Indicaciones: tumores T1 46\%, adenomas sin levantamiento $29 \%$, neoplasia residual local $23 \%$. La mayoría Ila + Ilc DET $26 \%$ vs. DET $50 \%$. Histología: 14 lesiones benignas, 21 lesiones malignas: $\mathrm{Cis} n=7$, $\mathrm{T} 1=12, \mathrm{~T} 2=2$ ), reportando éxito técnico DET $90 \%$ vs. DES $94 \%, p=0.999$; R0: DET $90 \%$ vs. DES $81 \%$, $p=0.785$; resección curativa DET $46 \%$ vs. DES $60 \%$, $p=0.670$. Eventos adversos DET 0 vs. DES $11 \%$ : tratamiento endoscópico 2/4, quirúrgico 2/4, $p<0.05$ ). Limitaciones: no se realizó seguimiento?.

\section{Disección endoscópica híbrida (DEH) para tratamiento de lesiones colorrectales}

McCarty $\mathrm{T}$, et al: revisión sistemática y metaanálisis de DES + REM para tratamiento de lesiones colorrectales (16 estudios: 2 ensayos clínicos, 4 prospectivos, 10 retrospectivos, $n=751$ ), reportando éxito técnico en $81.63 \%$ (72.07-88.44, IC 95\%, I2 83.36), R0 73.14\% (59.24-83.61, IC 95\%, I2 88.36), tamaño de resección $28.97 \mathrm{~mm} \pm 10.55 \mathrm{~mm}$, tiempo $48.88 \mathrm{~mm} \pm 22.37 \mathrm{~mm}$, eventos adversos $7.74 \%$ (hemorragia tardía $4.31 \%$, perforación 4.68\%), necesidad de cirugía 3.64\% (1.76\%$\left.7.37 \%, I^{2}=15.52\right)$ y recurrencia de $4.52 \%((1.40-13.65$, IC 95\%, I2 76.81). Limitaciones: No seguimiento ${ }^{8}$.

Yuen W, et al: retrospectivo unicéntrico, DET vs. REM + DET para tratar lesiones acorde a Paris en 62 pacientes $(\mathrm{H}$ 37, M 25, DET $n=33$ : Is 10 , Ila 7 , Ila + Ilc 5 , Ilb $3, \| l b+\operatorname{llc} 3$, Ilb + Ilc 3 , II c 3, NC 2 vs. REM + DET $n=29$ : Is 5, Ip 1, Ila 5, Ila + Ilc 7, llb 9, llb + Ilc 2). Resultados: DET vs. REM/ DET: éxito técnico $31 / 33$ (94\%) vs. $24 / 29(83 \%)$ ( $p>0.5)$; R0 30/31 (97\%) vs. 23/24 (96\%), $p>0.5$; y eventos adversos: DET 2/33 vs. REM + DES 0; $p>0.05)$; diámetro de resección: DET $19 \mathrm{~mm}$ (7 mm-40 mms) vs. REM + DET $36 \mathrm{~mm}$ (15 $\mathrm{mm}-60 \mathrm{~mm} ; \mathrm{p}<0.01)$. Limitaciones: retrospectivo9.

Brogyuk $\mathrm{N}$, et al: retrospectivo, unicéntrico, DES vs DES + REM (5 años, $n=42: H 27$, recto $n=37$, colon $=5$, edad media 65 años). Tipo de lesión: planas $15(36 \%)$, polipoideas $27(64 \%)$, tamaño $31 \mathrm{~mm}$ (10-80 mm), adenomas 17 (40.5\%) adenomas con displasia alto grado $12(28.5 \%)$, cáncer con invasión >sm1 $7(16.6 \%)$, Cis $3(7.1 \%)$, cáncer T2 $2(4.7 \%)$. DES vs. DES + REM: Resección en bloque 28/29 (95.6\%) vs. $7 / 13(53.8 \%), p>0.5$. R0 25 (86.7\%) vs. 7 (53.8\%) p>0.5; eventos adversos: Perforación 19/42 (45\%), 15 
tratadas con clips. 1 con OVESCO ${ }^{\text {TM }}, 1$ quirúrgicamente y 1 con cefalosporinas y hemorragia 1 , tratada con termocoagulación. Endoscopia de seguimiento 28/42 $(66.6 \%)$ con resultados negativos. Limitaciones: retrospectivo y seguimiento irregular ${ }^{10}$.

\section{Conclusiones}

- DES es eficaz y segura para lesiones colorrectales planas, elevadas o de extensión lateral, inclusive $>20$ $\mathrm{mm}$, de localización difícil y previamente tratadas, con baja tasa de recurrencia.

- Las lesiones de localización difícil tratadas con DES ameritan seguimiento endoscópico más estrecho (menor tasa de resección en bloque y mayor tasa de recurrencia local) respecto a las de otras localizaciones.

- DES es más eficaz en pólipos malignos de recto y sigmoides que del resto del colon.

- DET se reserva para lesiones no tratables por REM o DES, con equiparable tasa de éxito clínico y eventos adversos. La limitación de DET es el tamaño de la lesión (generalmente $<20 \mathrm{~mm}$ ).

- Las lesiones colorrectales más grandes y complejas pudieran ser tratados con DEH: una combinación de DES o DET para el componente invasor o sin levantamiento y REM o DES para el componente de extensión lateral. Se requieren estudios prospectivos que analicen esta modalidad terapéutica.

\section{Financiamiento}

Los autores no recibieron patrocinio para llevar a cabo este artículo.

\section{Conflicto de intereses}

Los autores declaran no tener conflicto de intereses alguno.

\section{Bibliografía}

1. Meier-Benjamin, Schmidt Arthur, Küllmer Armin et al: Efficacy and safety of Endoscopic Full-Thickness Resection with the FTRD in the colorectum: evaluation of a large PMCF Analysis. Presentado en cartel virtual durante la DDW; 2020 Mayo 2-5. ePoster 874-2020.

2. Ghersi Stefania, Gazzola Alesia, Landi Stefano et al: Advanced endoscopic resection techniques for local recurrent colorectal adenomas: A single center prospective study. Presentado en cartel virtual durante la DDW; 2020 Mayo 2-5. ePoster Mo1699-2020.

3. Stravropoulos Stravos N., Zhang Xiaocen, Ly Erin K, et al: Endoscopic submucosal disection for colorectal cancer and adenoma: A large single-operator cohort from the USA with long-term follow-up. Presentado en cartel virtual durante la DDW; 2020 Mayo 2-5. ePoster Mo1750-2020.

4. Lacopini Federico, Grossi Cristina, Lucidi Cristina et al: Long-term outcomes of colorectal endoscopic submucosal dissection in difficul locations: Dentate line, ileocecal valve and cecum. Presentado en cartel virtual durante la DDW; 2020 Mayo 2-5. ePoster Mo1753-2020.

5. Uchima Hugo, Herreros de Tejeda Alberto, Ramos-Zavala Felipe et al: Endoscopic submucosal dissection for colorectal (CR-ESD) malignant polyps. Results of a prospective multicenter western cohort. Presentado en cartel virtual durante la DDW; 2020 Mayo 2-5. ePoster 361-2020.

6. Bazarbashi Ahmad Najdat, McCarty Thomas R., Dolan Russel D. et al: Endoscopic full-thickness resection of colorectal lesions: A systematic review and meta-analysis. Presentado en cartel virtual durante la DDW; 2020 Mayo 2-5. ePoster Mo1714-2020.

7. Suchanek Stepan, Falt Premysi, Ngo Ondrej et al: Endoscopic full-thickness resection vs. endoscopic submucosal dissection in colorectal neoplasia therapy- Bicentric prospective randomized study. Presentado en cartel virtual durante la DDW; 2020 Mayo 2-5. ePoster Mo1630-2020.

8. McCarty Thomas R., Bazarbashi Ahmad Najdat, Thompson Christopher C., Aihara Hiroyuki: Hybrid endoscopic submucosal dissection for treatment of colorectal lesions: A systematic review and meta-analysis. Presentado en cartel virtual durante la DDW; 2020 Mayo 2-5. ePoster Mo1758-2020.

9. Yuen William, Mahadev SriHari, Yuen Poi Yu Sofia et al: Hybrid technique using endoscopic mucosal resection and endoscopic full-thicnkess resection for large colorectal lesions: Initial north american experience. Presentado en cartel virtual durante la DDW; 2020 Mayo 2-5. ePoster Sa2022-2020.

10. Brogyuk Nagyija, Ngo Ondrej, Zavoral Miroslav, Suchaneck Stepan: Efectiveness of endoscopic submucosal dissection alone and in combination wiht endoscopic mucosal resection (Hybrid technique) in colorectal neoplasia therapy. Presentado en cartel virtual durante la DDW; 2020 Mayo 2-5. ePoster Mo1684-2020. 